\title{
Shape effect of nanoparticles on tumour penetration in monolayers versus spheroids
}

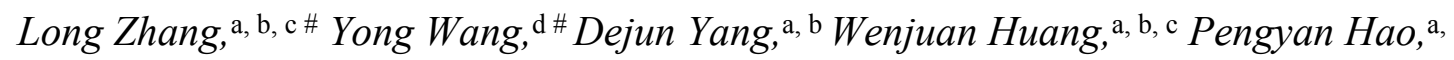
b, c Sheng Feng, ${ }^{\mathrm{e}}$ Dietmar Appelhans, ${ }^{\mathrm{f}}$ Tinghong Zhang, ${ }^{\mathrm{b}, \mathrm{c} *}$, Xingjie Zan, ${ }^{\mathrm{a}, \mathrm{b}, \mathrm{c} *}$

a School of Ophthalmology and Optometry, Eye Hospital, School of Biomedical Engineering, Wenzhou Medical University, Wenzhou, Zhejiang Province, 325035, PR China.

${ }^{\mathrm{b}}$ Wenzhou Institute of Biomaterials and Engineering, CNITECH, Chinese Academy of Sciences, Wenzhou, Zhejiang Province, 325001, PR China.

c Engineering Research Center of Clinical Functional Materials and Diagnosis \& Treatment Devices of Zhejiang Province, Wenzhou Institute of Biomaterials and Engineering, CAS Wenzhou, Zhejiang Province, 325001, PR China

${ }^{\mathrm{d}}$ Institute of Materials Research and Engineering, Fusionopolis Way, Innovis, 138634, Singapore.

e Department of Pathology and Laboratory Medicine, Hospital of the University of Pennsylvania, Philadelphia, Pennsylvania, 19107, USA.

${ }^{\mathrm{f}}$ Leibniz Institute of Polymer Research Dresden, Hohe Straße 6, 01069 Dresden, Germany.

\# These authors contributed equally to this paper. 
* Tinghong Zhang, Tel: +86 577 8017519, E-mail: zhangth@wibe.ac.cn; Xingjie Zan, Tel: +86 577 8017519, E-mail: xjzan2000@hotmail.com.

(a)

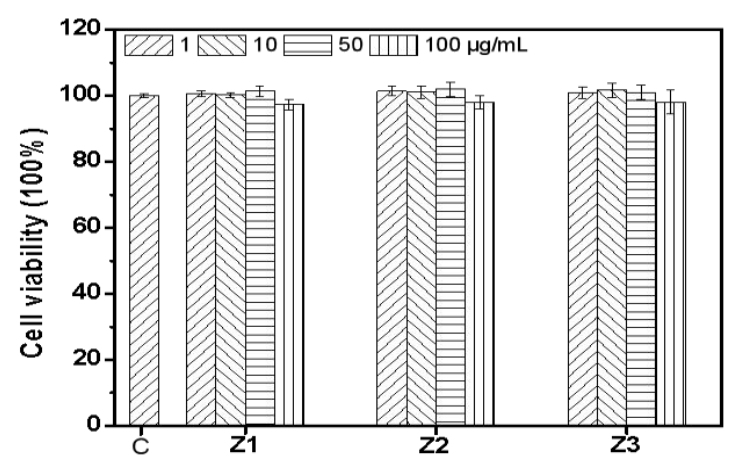

(b)

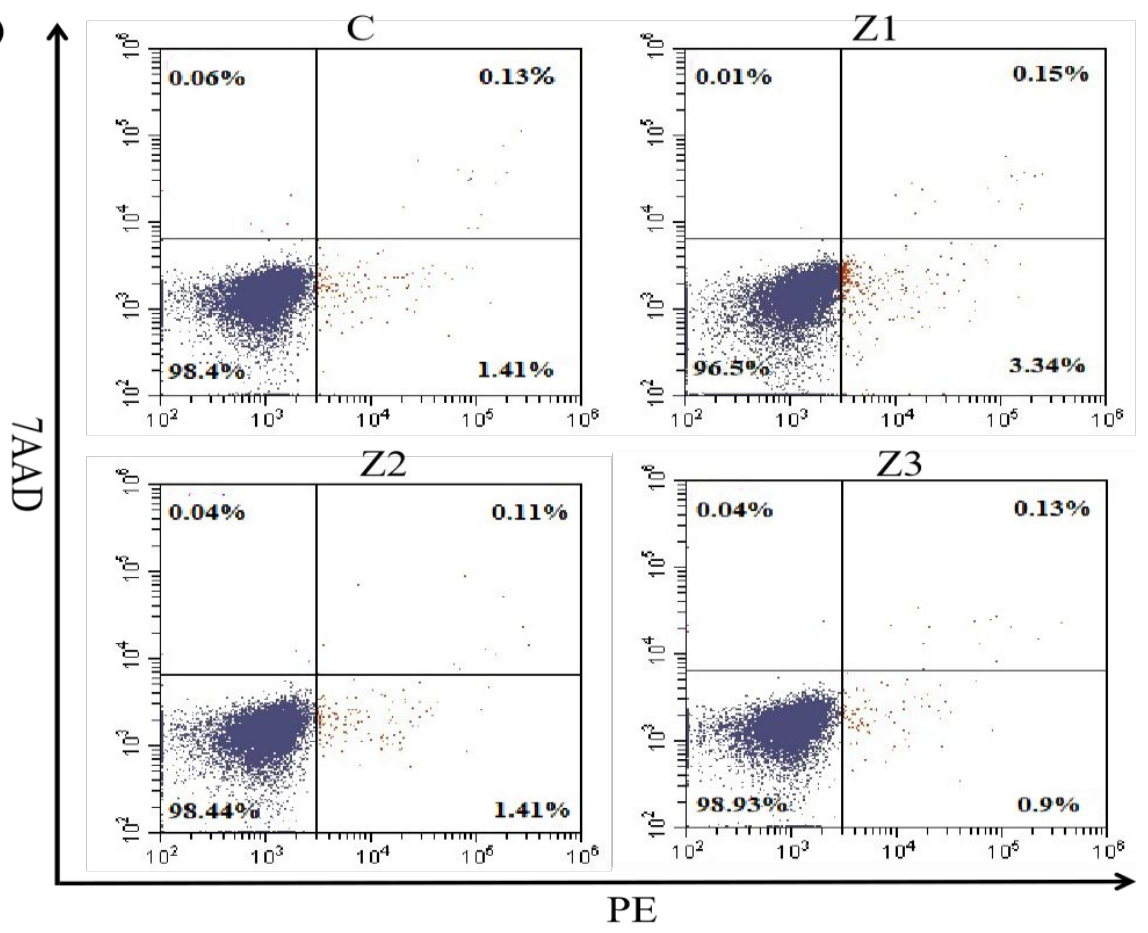

Figure. S1 (a) The viability tested by CCK-8 assay after incubating HeLa monolayer cells with particles (Z1, Z2 and Z3) at various concentrations for $24 \mathrm{~h}$. (b) Representative image of apoptosis assay by flow cytometry. Hela cells were treated with $10 \mu \mathrm{g} / \mathrm{mL}$ different samples for $72 \mathrm{~h}$. The apoptosis cells were assessed by flow cytometry assay. Z1 represents the spherical PS modified with PPI-G4. Z2 and Z3 represent stretched $\mathrm{Z1}$ with $\mathrm{AR} 2.8$ and 7.5 respectively. C: medium treated cells. 

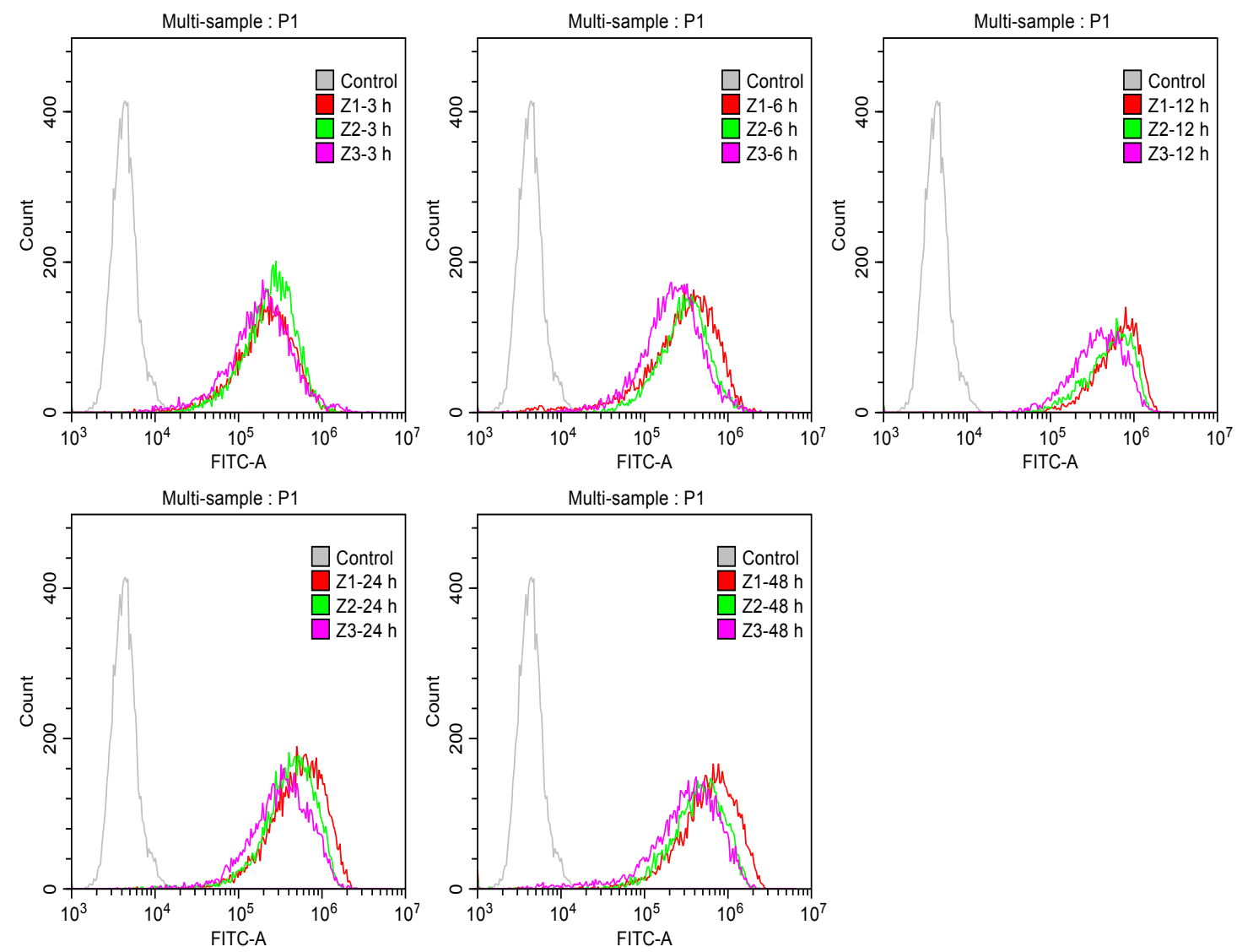

Figure. S2 Representative image of cellular uptake by flow cytometry. Hela cells were treated with $10 \mu \mathrm{g} / \mathrm{mL}$ different samples for indicated times $(3,6,12,24$ and 48 h). Cellular uptake were assessed by flow cytometry assay. Control: medium treated cells. 


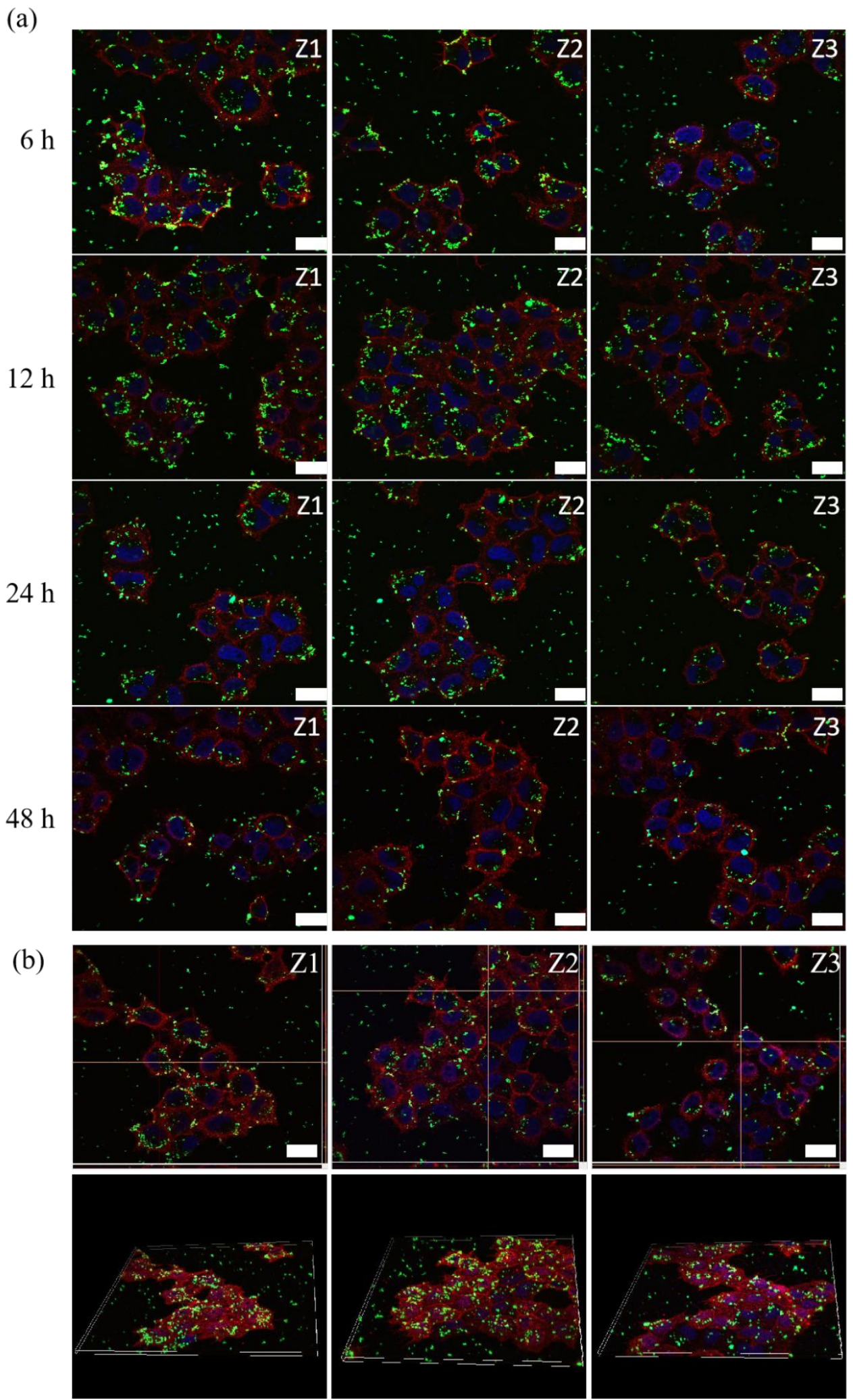

Figure. S3 (a) CLSM images of monolayer HeLa cells after incubation for 6, 12, 24 and $48 \mathrm{~h}$ with particles. (b) The confocal fluorescence images were reconstructed with both the orthographic view (above) and the 3D view (below). The cell membrane was 
stained with WGA conjugated with Alexa Fluor 555 (red), and the cell nucleus with Hoechst (blue), poly(styrene) were labeled with FITC (green). Z1 represents the spherical PS midified with PPI-G4. Z2 and Z3 repesent stretched Z1 with AR 2.8 and 7.5 respectively. scales bar: $25 \mu \mathrm{m}$.

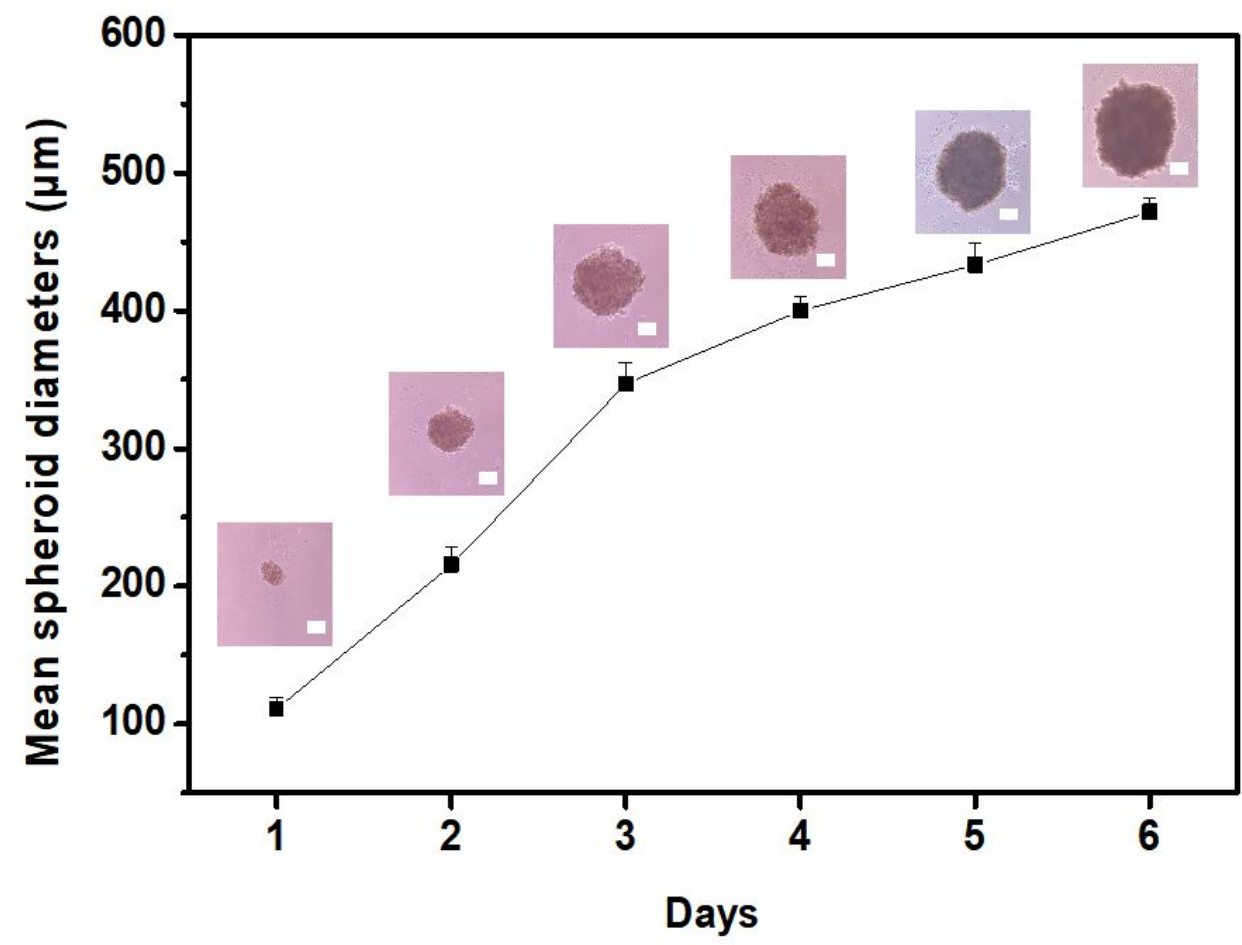

Figure. S4 Growth curve of spheroids and representative images of HeLa spheroids at $1,2,3,4,5$ and $6 \mathrm{~d}$ post centrifugation. The scales bar represent $100 \mu \mathrm{m}$. 

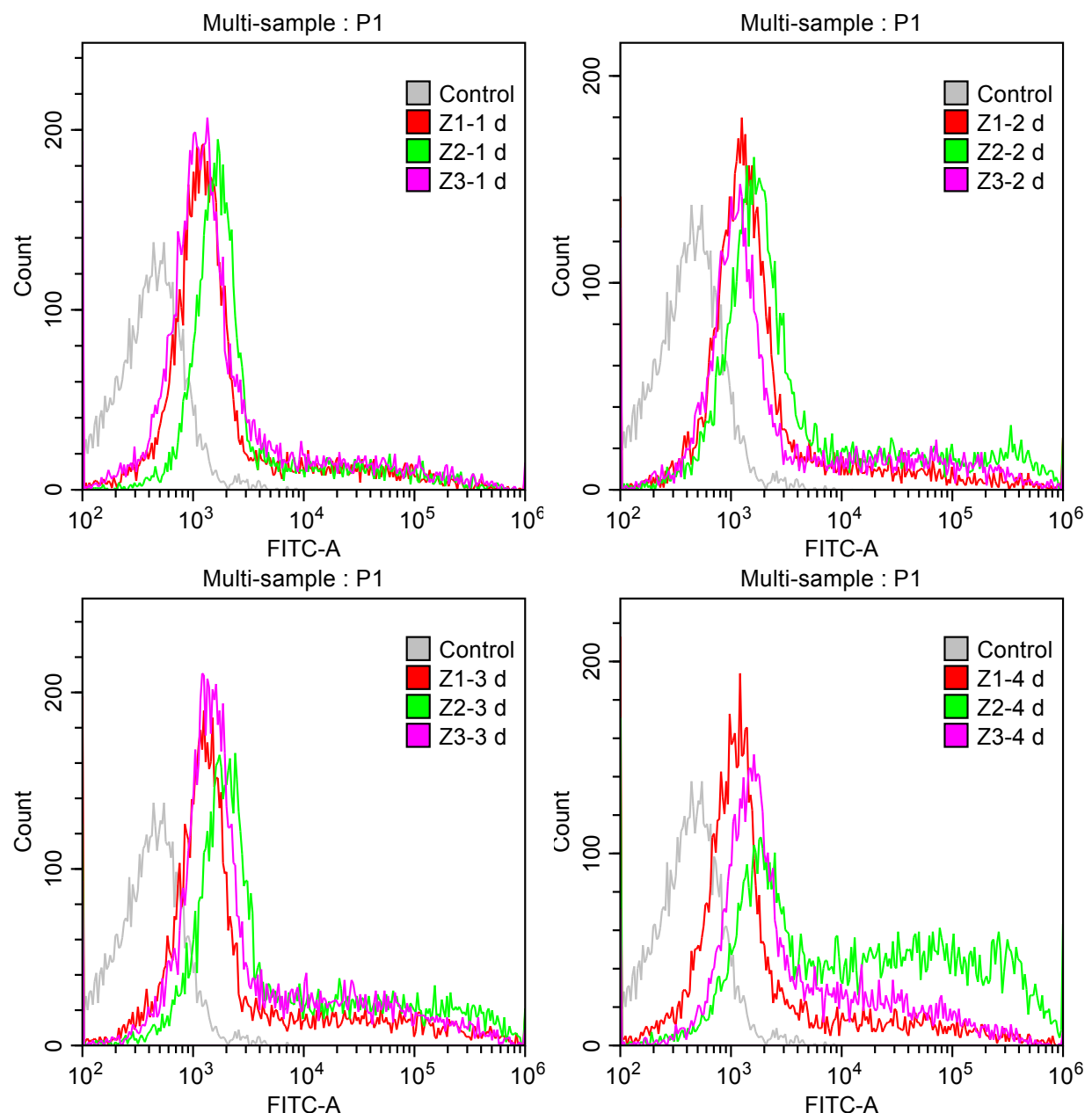

Figure. S5 Representative image of cellular uptake by flow cytometry. 3D spheroids were treated with $10 \mu \mathrm{g} / \mathrm{mL}$ different samples for indicated times $(1,2,3$ and $4 \mathrm{~d})$. The fluorescence positive cells were assessed by flow cytometry assay. Control: medium treated cells. 

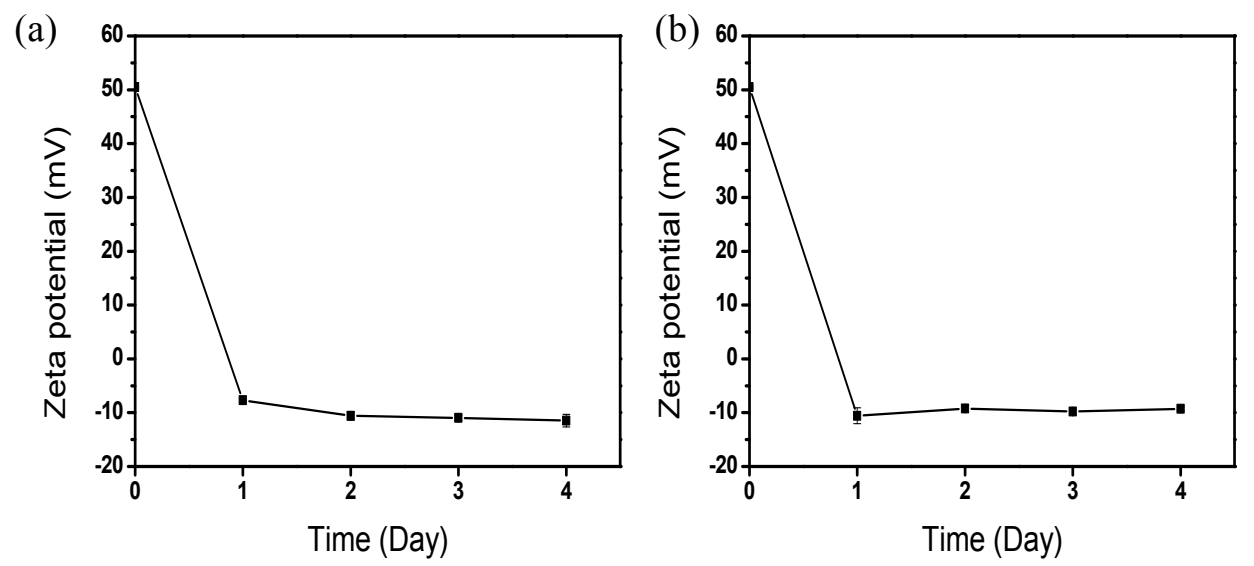

Figure. S6 Zeta potentials of Z1 after incubated with DMEM medium with fetal bovine serum $(10 \%, v / v)(a)$, or PBS with $50 \%$ fetal bovine serum (b) for indicated days. 